\title{
Qu'est-ce qu'un fait de genre syntaxique ? Enjeux et fondements d'une catégorie descriptive à partir des lettres de Mme de Sévigné
}

\author{
Lignereux, Cécile \\ RARE (Rhétorique de l'Antiquité à la Révolution) Université Stendhal - Grenoble 3 \\ cecilelignereux@yahoo.fr
}

\begin{abstract}
Perçu à la manière d'une évidence intuitivement sensible, le goût affiché de Mme de Sévigné ${ }^{1}$ pour des tournures syntaxiques pourtant régulièrement condamnées au cours du siècle, et d'ailleurs destinées à se raréfier puis à disparaître quelques dizaines d'années plus tard, n'a pas manqué d'être signalé comme en témoigne l'avis d'É. Gérard-Gailly :

[...] elle écrivait comme en 1630, quand on n'était pas un écrivain de profession ; elle écrivait la langue qu'elle avait apprise dans un milieu tout bourgeois et un peu retardataire. Si elle eut, comme elle disait, la connaissance des beaux styles, elle ne songeait pas à s'en forger un elle-même ni à posséder la grammaire nouvelle ou prochaine, qu'elle ne confondait probablement pas avec le style. Elle ne «parlait» pas Vaugelas. ${ }^{2}$
\end{abstract}

Certes, l'expressivité syntaxique de la prose sévignéenne résulte en grande partie d'une force d'inertie linguistique, la marquise persistant à employer des tournures vieillies - c'est-à-dire préférant conserver ses usages syntaxiques, même voués à une élimination progressive, plutôt que de se plier à des injonctions normatives qui ne jouissent à ses yeux d'aucune autorité ${ }^{3}$. Pourtant, force est de constater que la syntaxe volontiers débridée des lettres de Mme de Sévigné n'a pas encore fait l'objet d'une étude d'ensemble propre à justifier des jugements qui, s'ils ne sont pas faux, reposent davantage sur des constats impressionnistes que sur des relevés méthodiques. Dès lors, il s'agit d'expliquer pourquoi et comment certaines configurations doivent être envisagées comme autant de composantes d'un authentique «style syntaxique ${ }^{4}$ parfaitement reconnaissable. Dans la mesure où les tournures qui paraissent vivantes ou pittoresques aux yeux des lecteurs d'aujourd'hui ne méritent assurément pas d'être prises en compte au même titre, quelles catégories descriptives adopter? Afin de ne pas reproduire l'erreur méthodologique qui consiste à faire l'inventaire de toutes les constructions qui nous semblent exotiques (et qui ne semblent telles qu'à proportion de notre méconnaissance de la langue classique $)^{5}$, il paraît nécessaire à la fois de recourir à la dichotomie entre faits de langue et faits de style et de dépasser la radicalité d'une telle opposition en réservant une troisième rubrique aux faits de genre. Reposant sur « une conception continuiste de la langue au style » qui permet d'interpréter avec prudence la singularité des faits syntaxiques isolés ponctuellement, cette tripartition présente l'avantage de postuler une gradualité entre les faits de langue (qui ne font qu'illustrer la souplesse et la diversité variationnelle de la langue classique), les faits de genre (qui sont surtout liés au cadre communicationnel de la lettre familière) et les faits de style (dont la convergence fait émerger de façon 
concomitante sens et valeur), envisagés comme autant de «saisies » de «l'appropriation de la langue $»^{6}$. Si le repérage des faits de langue et des faits de style est relativement aisé, il n'en va pas de même des faits de genre, en raison précisément de leur caractère intermédiaire. C'est ce qu'illustre la démarche qui permet de repérer, de décrire et d'interpréter comme fait de genre l'un des phénomènes syntaxiques les plus saillants de la correspondance : la gestion de l'anaphore pronominale.

Indéniablement, Mme de Sévigné ne se montre guère soucieuse d'éviter les hésitations que suscite un maniement plein de souplesse de la référence pronominale en général, et des pronoms personnels en particulier. Ce n'est pas un hasard si l'usage de ces derniers a fait l'objet de nombreuses corrections de la part d'éditeurs plus attentifs au confort de leurs lecteurs que de fidélité au texte autographe ${ }^{7}$. On n'en finirait pas de citer les cas où l'identification du référent de l'expression anaphorique, c'est-à-dire la localisation de son antécédent, n'est pas immédiate. Plutôt que de multiplier les exemples où l'aperception de la chaîne anaphorique sollicite autant la vigilance que la sagacité du lecteur (notamment lorsque l'antécédent du pronom est distant de plusieurs lignes), contentons-nous de citer trois cas extrêmes, où la compréhension engage de périlleux mécanismes interprétatifs, tellement irréductibles à la grammaire que l'éditeur a utilement précisé en note la résolution de l'énigme référentielle :

J'avais été dîner le vendredi à Pomponne, où Mme de Vins reçut une lettre de vous. Nous causâmes fort sur votre sujet ; je suis fort contente de la manière dont elle s'intéresse à tout ce qui vous touche. M. de Pomponne la gronda fort de ne vous avoir point parlé de lui dans ses lettres; ce fut une fort jolie querelle. Elles seront encore là quinze jours. (8 novembre $1679:$ II, 728$)^{8}$

J'en fais mes compliments à $\mathrm{M}$. de Grignan et à Monsieur le Coadjuteur et à vous, et je recommence à vous en faire de tous ceux que je vous ai déjà nommés, qui m'ont repriée : le Bien Bon, mon fils qui va encore écrire à M. de Grignan. Il part demain. (23 février $1680:$ II, 844) ${ }^{9}$

J'ai déjà demandé à mes amies tous les secours qu'ils nous ont déjà donnés. (23 avril $1690:$ III, 872) $)^{10}$

Vous avez une capacité sur les affaires qui me surprend. On peut avoir beaucoup d'esprit sans en avoir de cette sorte. Je l'admire d'autant plus qu'il est cent piques au-dessus de ma tête ; vous savez ceux dont je me servais. Enfin vous en avez de toutes les façons. Remerciez-en Dieu, car assurément ce n'est pas de vous que viennent tous ces dons. Quand une belle et aimable femme les a reçus du Ciel, comme vous, c'est une merveille. J'en conviens, on leur permettait quelquefois de n'être point habiles. (21 mai $1690:$ III, 877)

Alors que les obscurités créées par l'emploi peu rigoureux des termes anaphoriques sont minutieusement traquées par les Remarqueurs et par les critiques, qui prennent un malin plaisir à épingler les approximations contraires à la clarté, l'épistolière fait preuve de la plus grande liberté en la matière. Certes, Mme de Sévigné se montre sourde aux injonctions de netteté - Vaugelas ayant accrédité l'idée selon laquelle « le plus grand des vices contre la netteté, ce sont les equivoques, dont la plus-part se forment par les pronoms relatifs, demonstratifs et possessifs $»^{12}$. Alors que pour Vaugelas et ses successeurs, l'«approche de l'anaphore est [...] régie par les deux principes de netteté (un 
pronom doit avoir un antécédent clairement identifiable) et de proximité (cet antécédent est le GN le plus proche) $\gg^{13}$, la marquise ne s'embarrasse guère de scrupules concernant les ambiguïtés référentielles. Pourtant, ce serait tomber dans l'écueil de la surinterprétation que d'y voir une désinvolture à caractère proprement idiolectal, pour deux raisons notamment. La première est d'ordre linguistique : les ambiguïtés référentielles résultant d'un maniement des pronoms imperméable à la normalisation prônée par les grammairiens de l'époque subsistent encore couramment, bien au-delà du seul XVII ${ }^{\mathrm{e}}$ siècle $^{14}$. La seconde est d'ordre générique : dans la mesure où l'épistolière fait confiance en une compréhension à demi-mot permise par une authentique relation de familiarité, elle n'éprouve pas le besoin d'être plus claire. D'ailleurs, il faudrait être de mauvaise foi pour affirmer que les passages qui paraissent embrouillés au lecteur d'aujourd'hui nuisent à l'intelligibilité du message ! Nous nous contenterons d'un seul exemple, où le pronom de reprise est éloigné du nom propre qui lui fournit sa référence :

Nous parlions de vous l'autre jour, Mme de La Fayette et moi, et nous trouvâmes qu'il n'y avait au monde que Mme de Rohan et Mme de Soubise qui fussent ensemble aussi bien que nous sommes. Et où trouverez-vous une fille qui vive avec sa mère aussi agréablement que vous faites avec moi ? Nous les parcourûmes toutes. En vérité, nous vous fîmes bien de la justice, et vous auriez été contente d'entendre tout ce que nous disions. Il me paraît qu'elle a bien envie de servir M. de Grignan [...]. (20 octobre 1679 : II, 711) $)^{15}$

Alors que l'éloignement (d'un point de vue textuel, et non cognitif) de l'antécédent appelle une note de la part d'un éditeur soucieux d'épargner au lecteur la reconstitution rétrospective de l'enchaînement anaphorique, il est certain que ce genre de processus, caractéristique d'une continuité thématique seulement interrompue de manière superficielle, ne posait absolument aucun problème à Mme de Grignan - habituée aux phénomènes de substitution pronominale les plus (grammaticalement) opaques, et parfaitement au courant de tout ce à quoi sa mère fait allusion. Les pratiques anaphoriques de Mme de Sévigné ne sont donc pas à envisager comme des déviances ou des infractions ${ }^{16}$ à l'égard de règles à la fois naissantes et sans conséquences dans une conversation entre mère et fille.

À y regarder de près en effet, les trois types d'obstacles qui entravent l'accès au référent des termes anaphoriques ne font que refléter la permanence d'usages encore prégnants à la fin du siècle - usages d'autant moins étonnants qu'ils sont le fait d'une épistolière déjà âgée et qui s'exprime sur le mode de la conversation. C'est pourquoi nous nous contenterons de résumer les riches analyses déjà disponibles sur le maniement des procédés anaphoriques dans les lettres de Mme de Sévigné.

Premièrement, les problèmes de clarté dans l'accès au référent peuvent être posés par la distance entre le marqueur anaphorique et son antécédent - puisqu'il est désormais admis que «le marqueur anaphorique doit être rapproché de son antécédent $\rangle^{17}$. En fait, seuls y trouveraient à redire soit des Remarqueurs qui, hantés par l'idéal de clarté, sont entraînés à débusquer les plus légères ambiguïtés ${ }^{18}$, soit des lecteurs d'aujourd'hui, habitués à avoir « la mémoire courte ${ }^{19}$ au point d'être dérangés par des relations anaphoriques spatialement lointaines. À l'évidence, Mme de Sévigné, à la fois coutumière 
d'un maniement de l'anaphore très lâche, confiante dans l'intelligence complice de sa destinataire, et heureuse de pouvoir laisser courir sa plume aussi vite que ses pensées dans le cadre d'un commerce familier, n'a aucune raison de s'inquiéter du degré d'intelligibilité de ses reprises pronominales.

Deuxièmement, les ambiguïtés référentielles peuvent provenir de la «concurrence entre plusieurs antécédents possibles pour un terme anaphorique $»^{20}$. En réalité, cette concurrence n'existe que pour le lecteur mal intentionné ou inattentif. Dans la grande majorité des cas, la compréhension s'opère très rapidement. Il serait absurde de reprocher à Mme de Sévigné de manquer de netteté dans l'emploi des anaphoriques, alors que l'objet du discours est, dans l'esprit des correspondantes, parfaitement clair.

Troisièmement, l'accès au référent peut être gêné par la «non-coréférence de deux occurrences successives d'un même marqueur anaphorique $"^{21}$ - dont le cas typique est celui de l'anaphore d'un nom à déterminant zéro, éclairé de manière décisive par I. Landy-Houillon ${ }^{22}$. Certes, la marquise, en femme d'esprit dotée d'une sensibilité aiguë aux phénomènes linguistiques, a du goût pour les formes d'ingéniosité que constituent d'une part, l'utilisation désinvolte et provocante des anaphoriques ${ }^{23}$ et d'autre part, le décryptage des énigmes référentielles occasionnées par le maniement acrobatique des pronoms - en particulier dans le cas où le "prélèvement de l'élément nominal hors de sa locution d'origine, puis sa représentation dans un autre membre du système » entrâne «la dislocation des phraséologies $»^{24}$. Pourtant, il faut garder à l'esprit que le phénomène d'anaphore non coréférentielle est très courant à l'époque ${ }^{25}$, et donc ne pas en tirer des conclusions hâtives sur les audaces syntaxiques de Mme de Sévigné.

Résumons la leçon méthodologique de ces quelques mises au point: ce n'est pas parce que nous sommes habitués au respect de règles destinées à guider l'accès au référent, et tout spécialement le référent d'un pronom anaphorique, qu'il faut surinterpréter les cas où la netteté de la référence paraît compromise. Tout dépend en effet du point de vue adopté : pour des Remarqueurs obsédés de clarté, le peu de rigueur qu'observe la marquise dans la construction de «chaînes de référence $»^{26}$ peut être stigmatisé comme équivoque ${ }^{27}$; en revanche, si l'on accepte de pratiquer une approche mémorielle, et non pas seulement textuelle, de l'anaphore en langue classique, on se rend compte que les énoncés ne recèlent pas d'ambigüité ${ }^{28}$. Ce n'est d'ailleurs pas un hasard si N. Fournier, au moment où elle analyse les cas de non-coréférence, recourt à une lettre de Mme de Sévigné à Mme de Grignan (12 février 1671 : I, 157-158) pour illustrer la nécessité de substituer au principe de proximité le principe de saillance $^{29}$, seul pertinent pour ne pas reconduire les erreurs de perspective - surtout en situation d'échange épistolaire familier. Si Mme de Sévigné ne s'embarrasse guère des ambiguités référentielles, il convient de se prémunir contre l'erreur de perspective qui consiste à juger de la fluidité de la syntaxe sévignéenne avec nos yeux de lecteurs habitués à appréhender l'articulation du sens et de la référence d'une manière sensiblement différente de celle des contemporains de la marquise. De fait, certains procédés de sémantique référentielle qui étaient alors courants risquent fort d'apparaitre comme des audaces stylistiques cultivées aux dépens de la clarté, tant les seuils de tolérance en matière de 
construction de la référence pronominale et d'identification du référent - c'est-à-dire de la «déontologie de l'anaphore ${ }^{30}$ - ont bougé depuis. Dès lors que l'on met en relation les prétendues équivoques non seulement avec la variation diachronique des modes d'appréhension du référent mais encore avec la situation de communication épistolaire, le peu de rigueur qu'observe Mme de Sévigné dans les mécanismes anaphoriques ne saurait être considéré comme un fait de style syntaxique propre aux lettres à Mme de Grignan : il suffit de se souvenir que les exigences de proximité textuelle sont trop nouvelles - la constitution des normes en matière de référence pronominale se fait de manière progressive tout au long du XVII ${ }^{\mathrm{e}}$ siècle ${ }^{31}$ - et trop étrangères à la connivence entre correspondantes intimes pour que Mme de Sévigné s’embarrasse d'ambiguïtés virtuelles.

Cependant, il ne faudrait pas exagérer la souplesse du maniement pronominal dans les lettres de Mme de Grignan, qui, s'il sacrifie souvent l'intelligibilité immédiate à la vitesse de l'écriture, n'est en rien comparable aux libertés d'un cardinal de Retz ou d'un duc de La Rochefoucauld, motivés par d'irréductibles postures auctoriales. D'ailleurs, lorsque Mme de Sévigné s'aperçoit que certaines anaphores pronominales risquent de poser à sa destinataire des problèmes d'identification, voire d'engendrer des confusions, elle prend soin d'orienter vers le bon référent. C'est ainsi qu'elle glose parfois des énoncés qui lui semblent ambigus ou peu clairs grâce à une reformulation paraphrastique ${ }^{32}$ introduite par « c'est-à-dire » - reformulation dont la dimension d'amplification est patente :

\footnotetext{
Monsieur de Grignan, Monsieur le Coadjuteur, vous faites bien de m'aimer ; je vous défie tous deux d'aimer mieux Mme de Grignan que moi, c'est-à-dire que je l'aime. (20 novembre $1673:$ I, 624) ${ }^{33}$

Vous ne m'expliquez que trop bien vos périls. Je ne les comprends pas, c'est-à-dire je ne comprends pas comme on s'y peut exposer. (2 juin $1672:$ I, 524)
}

De même, il lui arrive de préciser sa pensée lorsque le terme qui fournit sa référence à la reprise pronominale n'a pas été employé précédemment,

Songez-y, ma bonne, à ce départ, et ne le pressez point; vous en êtes la maîtresse. (printemps ou été 1679 : II, 669)

de prendre soin d'insister sur la continuité de son propos après un processus anaphorique audacieux lié à la rapidité de la pensée,

Je fus hier chez Mme de Verneuil, au retour de Saint-Maur, où je fus avec Monsieur le Cardinal. Je trouvai à l'hôtel de Sully Mlle de Lannoi, mariée au petitfils du vieux comte de Montrevel. La noce s'est faite là. Jamais vous n'avez vu une mariée si drue ; elle va droit à son ménage, et dit déjà «mon mari ». Il avait la fièvre, ce mari, et la devait avoir le lendemain ; il ne l'eut point. (31 mai 1675 : I, 720)

Il y a plus de bien que de mal à cette qualité docile, qui fait honneur à ce qui est bon, et qui est si propre à occuper agréablement certains temps de la vie. Enfin, ma fille, je vous la souhaiterais, cette qualité. (8 février 1690 : III, 833)

de gloser le processus anaphorique qui permet de passer du singulier au pluriel, 


\begin{abstract}
Ma bonne, je vous en fais aussi [des compliments] sur ce que vous êtes guérie de votre rhume. Je les crains, ces rhumes, parce qu'ils sont souvent accompagnés de votre mal de gorge [...]. (26 avril 1690 : III, 873)
\end{abstract}

d'éviter à sa lectrice de faire l'effort de se souvenir des interlocuteurs et des hôtes de sa mère,

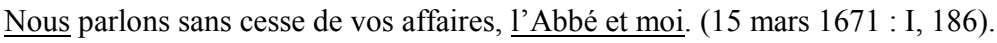

Nous en parlons, Monsieur d'Uzès et moi, et regardons les chagrins qui sont attachés à quelque résolution qu'on prenne. (13 janvier $1672: \mathrm{I}, 413)$

Il a interrompu ma lettre, ce bel abbé, et il m'a promis de faire si bien que je ne puis douter que nous n'ayons notre pension. (7 août 1676 : II, 363)

Nous avons dîné, le Chevalier, l'Abbé, Corbinelli et moi, et nous avons un peu essayé le cuisinier. (18 octobre $1679:$ II, 707)

Le voilà arrivé, ce fripon de Sévigné. (2 février 1680 : II, 828)

ou encore de neutraliser les ambiguïtés liées à la concurrence d'un autre référent grammaticalement possible :

Ma lettre vous trouvera, comme Dulcinée, dans l'agitation du mouvement de cette compagnie ; gardez-la, je dis ma lettre, et puis vous la lirez à loisir. (2 août 1671 : I, 311)

Je vous avoue que j'ouvrirai vos lettres de vendredi avec une grande impatience et une grande émotion. Mais elles ne sont pas d'importance, mes émotions, et un verre d'eau en fait l'office. (13 septembre $1671:$ I, 344)

Je l'aime [le Cardinal] et honore d'une manière à me faire un tourment de cette pensée ; le temps ne répare point de telles pertes. Il n'a fait, ce temps, jusqu'ici, qu'augmenter la tendresse et la sensibilité que j'ai pour vous [...]. (28 juillet 1677 : II, 505)

Elle [Mme de La Fayette] sera alerte sur les chevaliers de l'ordre et sur tout. Le mariage se fera dans un mois, malgré l'écrevisse, qui prend l'air tant qu'il peut, mais il sera encore fort rouge en ce temps-là. Elle prend, Mme de La Fayette, des bouillons de vipères, qui lui redonnent une âme. (20 octobre 1679 : II, 711)

Si cette structure s'apparente au procédé de la dislocation, le pronom étant redoublé par le groupe nominal ou le nom propre qui vient en expliciter la référence, elle doit pourtant être interprétée moins comme un moyen stylistique d'emphase, que comme une retouche corrective, voire une rectification, qui résulte de la prise de conscience, par l'épistolière, d'un manque de clarté que vient justement résoudre le constituant détaché en rappel - bref, comme un dispositif syntactico-énonciatif visant à la désambiguïsation. Certes, l'élément détaché à droite reçoit un accent d'insistance. Mais loin d'être motivée par des préoccupations esthétiques d'ordre rythmique, la dislocation apparaît surtout comme le moyen d'élucider un pronom employé de manière intempestive :

Mais savez-vous bien ce qu'elles étaient devenues ces chères lettres que j'attends et que je reçois avec tant de joie ? (21 juin $1671: \mathrm{I}, 274)$

Je vous assure qu'il est fort à plaindre, ce pauvre petit frère ; il est accablé de maux et de remèdes. (22 septembre 1680 : III, 21)

Il est revenu au gîte, ma chère enfant, ce paquet égaré. (12 août 1689 : III, 665)

En vérité, elle est trop grande et trop sensible, cette amitié [...]. (23 avril 1690 : III, 868)

Je le crois part, ce pauvre Marquis, avec tous les autres. (26 avril $1690:$ III, 874) 
De telles structures, qui importent dans la lettre des phénomènes de reprise et de reformulation particulièrement fréquents dans la langue orale, attestent que l'épistolière, même si elle écrit au fil de ses pensées, se montre attentive à sa destinataire. Certes, une telle démarche n'est pas la plus courante dans la correspondance. Néanmoins, il faut retenir le souci que manifeste parfois Mme de Sévigné d'éclairer la référence de pronoms qui font surgir plusieurs antécédents potentiels - ce qui invite à ne pas se méprendre sur la négligence des tours anaphoriques en les imputant par exemple à une désinvolture ludique et provocante, interprétable en termes de choix voire d'intention stylistiques. Si la représentation pronominale n'est manifestement pas astreinte aux idéaux de clarté, de pureté et de netteté vantés par les Remarqueurs, c'est tout simplement parce que Mme de Sévigné n'éprouve ni l'impression d'être confuse, ni le besoin d'être plus claire - la situation de communication propre à la lettre familière dispensant d'une scrupuleuse régularité.

Pour ne pas sous-estimer (en les réduisant à de simples faits de langue qui ne seraient que le symptôme de la diversité variationnelle propre à l'idiome classique) ni sur-évaluer (en les traitant comme des faits de style motivés par une volonté pragmatiquement orientée de ne pas respecter les nouvelles injonctions grammairiennes) certains phénomènes syntaxiques récurrents, il convient donc de ménager une place aux faits de genre, envisagés comme des usages linguistiques certes encore prégnants et relativement bien tolérés, mais dont l'apparition est non seulement permise mais encore favorisée par le cadre communicationnel - ce qui implique de substituer, au moins momentanément, à l'interprétation des effets esthétiques une réflexion sur leurs conditions de possibilité pragmatiques.

\section{Références bibliographiques}

\section{Bibliographie primaire}

Mme de Sévigné, Correspondance (1646-1696), Duchêne Roger (éd.), Paris, Gallimard, coll. « Bibliothèque de la Pléiade », 1972-1978.

Mme de Sévigné, Lettres de Mme de Sévigné, de sa famille et de ses amis, Monmerqué M. (éd.), Paris, Hachette, coll. «Les Grands Écrivains de la France », 1866, 14 tomes.

Mme de Sévigné, Lettres de Mme de Sévigné, Gérard-Gailly Émile (éd.), Paris, Gallimard, coll. « Bibliothèque de la Pléiade », 3 tomes, 1953-1957.

\section{Bibliographie secondaire}

Ayres-Bennett Wendy et Caron Philippe, «Écriture d'auteur, norme et variation au XVII ${ }^{\mathrm{e}}$ siècle : de la syntaxe et du style », dans Ayres Bennett Wendy et O'Donovan Patrick (dir.), Syntax and the literary system: New approaches to the interface between literature and linguistics, Cambridge, Cambridge French Colloquia, 1995, p. 71-91.

Ayres-Bennett Wendy et Caron Philippe, «Introduction», dans Les Remarques de l'Académie française sur le Quinte-Curce de Vaugelas (1719-1720). Contribution à une histoire de la norme grammaticale et rhétorique en France, Ayres-Bennett Wendy et Caron Philippe (éd.), Paris, 
Presses de l'École normale supérieure, coll. «Études \& Documents en Histoire de la langue française », 1996, p. 9-49.

Corblin Francis, Les formes de reprise dans le discours. Anaphores et chaînes de référence, Rennes, P.U.R., coll. « Langue/discours », 1995.

Fournier Nathalie, Grammaire du Français classique, Paris, Belin, coll. « Lettres Sup », 1998.

Fournier Nathalie, "Quelques problèmes concernant l'anaphore et les marqueurs anaphoriques dans Nicomède », L'Information grammaticale, $\mathrm{n}^{\circ} 76$, janvier 1998, p. 25-28.

Fournier Nathalie, «Norme et usage de l'anaphore pronominale en français classique : principe de proximité et principe de saillance du référent », dans BAUDRY Janine et CARON Philippe (dir.), Problèmes de cohésion syntaxique de 1550 à 1720, op. cit., p. 191-214.

Gülich Elisabeth et Kotschi Thomas, «Les Marqueurs de reformulation paraphrastique », Cahiers de Linguistique française, « Connecteurs pragmatiques et structure du discours. Actes du $2{ }^{\text {ème }}$ colloque de Genève (7-9 mars 1983) », Roulet Eddy (dir.), n 5, 1983, p. 305-351.

Jaubert Anna, " «La représentation pronominale. Contours et entours de la référence », L'Information grammaticale, $\mathrm{n}^{\circ} 82$, juin 1999, p. 29-37.

Jaubert Anna, «La diagonale du style. Étapes d'une appropriation de la langue », Pratiques, $\mathrm{n}^{\circ} 135$, "Questions de style », Petitjean André et Rabatel Alain (dir.), décembre 2007, p. 47-62.

Landy-Houillon Isabelle, «L'ellipse : une figure chez Mme de Sévigné », Littératures classiques, $\mathrm{n}^{\circ}$ 28, automne 1996, p. 285-294.

Landy-Houillon Isabelle, «Entre lexique et syntaxe : des interférences de la phrase et du pronom au XVII ${ }^{\mathrm{e}}$ siècle », dans Gross Maurice (dir.), La Lingua francese nel Seicento, Bari-Paris, AdriaticaNizet, «Quaderni del Seicento francese », 1989, p. 63-78.

Lignereux Cécile, «Les caprices syntaxiques de Mme de Sévigné : une épistolière sourde aux prescriptions ? », dans S. Branca-Rosoff, J.-M. Fournier, Y. Grishpun et A. Régent-Susini (dir.), Langue commune et changements de normes, Paris, Champion, coll. "Linguistique historique ", 2011, p. 321-332.

Murat Michel et Cartier-Bresson Bernard, «C'est-à-dire ou la reprise interprétative », Langue française, $\mathrm{n}^{\circ}$ 73, « La reformulation du sens dans le discours », Riegel Martin et Tamba Irène (dir.), février 1987, p. 5-15.

Reichler-Béguelin Marie-José, « Norme et Textualité. Les procédés référentiels considérés comme déviants en langue écrite », dans Schoeni Gilbert, Bronckart Jean-Paul et Perrenoud Philippe (dir.), La Langue française est-elle gouvernable ?, Neuchâtel-Paris, Delachaux et Niestlé, 1988, p. 185216.

Siouffi Gilles, Le "génie de la langue française" à l'âge classique. Recherches sur les structures imaginaires de la description linguistique de Vaugelas à Bouhours, thèse de doctorat, Cahné Pierre (dir.), 2 volumes, Université de Paris IV-Sorbonne, janvier 1995.

Siouffi Gilles, «Le regard des grammairiens », dans Combettes Bernard (dir.), Évolution et variation en français préclassique, Études de syntaxe, Paris, Champion, 2003, p. 19-67.

Sommier Édouard, «Introduction grammaticale », dans Mme de Sévigné, Lettres de Mme de Sévigné, de sa famille et de ses amis, Monmerqué M. (éd.), Paris, Hachette, coll. " Les Grands Écrivains de la France », 1866, tomes 13, p. XI-LXXXIV.

\footnotetext{
${ }^{1}$ Les citations des lettres de Mme de Sévigné, données entre parenthèses au fil du texte, mentionnent la date de la lettre et sa pagination (tome et page) dans l'édition de référence: Correspondance, éd. R. Duchêne, Paris, Gallimard, «Bibliothèque de la Pléiade », 1978.

2 É. Gérard-Gailly, «Introduction », Lettres de Mme de Sévigné, éd. É. Gérard-Gailly, Paris, Gallimard, 19531957 , t. I, p. 7-9.

${ }^{3}$ C. Lignereux, «Les caprices syntaxiques de Mme de Sévigné : une épistolière sourde aux prescriptions ? », dans S. Branca-Rosoff, J.-M. Fournier, Y. Grishpun et A. Régent-Susini (dir.), Langue commune et changements de normes, Paris, Champion, 2011, p. 321-332.

${ }^{4}$ Considérant que "la syntaxe est un des niveaux caractéristiques d'une écriture », W. Ayres-Bennett et de $\mathrm{Ph}$. Caron proposent de parler d'un «"style syntaxique" » qui « ne se laisse bien appréhender que si l'on accumule des connaissances sur le comportement syntaxique de l'époque dans laquelle s'insère notre auteur» («Écriture d'auteur, norme et variation au XVII ${ }^{\mathrm{e}}$ siècle : de la syntaxe et du style », dans W. Ayres-Bennett et P. O'Donovan
} 
(dir.), Syntax and the literary system: New approaches to the interface between literature and linguistics, Cambridge, Cambridge French Colloquia, 1995, p. 72).

${ }^{5}$ Si l'«Introduction grammaticale » d'É. Sommer, d'une indéniable richesse, recense la plupart des difficultés syntaxiques auxquelles peut se trouver confronté le lecteur, elle pèche par un empirisme pédagogique patent (Lettres de Mme de Sévigné, de sa famille et de ses amis, éd. M. Monmerqué, Paris, Hachette, coll. « Les Grands Écrivains de la France », 1866, 14 tomes, p. XI-LXXXIV).

${ }^{6}$ Nous faisons nôtre la terminologie d'A. Jaubert, «La diagonale du style. Étapes d'une appropriation de la langue », Pratiques, n¹35, «Questions de style », Petitjean A. et Rabatel (dir.), décembre 2007, p. 47-62.

${ }^{7}$ Sur la manière dont le chevalier Perrin, qui « recherche la clarté », « supprime ce qui est trop allusif et remplace un pronom par son antécédent », voir R. Duchêne, « Note sur le texte », dans Mme de Sévigné, Correspondance, éd. R. Duchêne, Paris, Gallimard, « Bibliothèque de la Pléiade », 1978, t. I, p. 797.

${ }^{8}$ R. Duchêne précise que le pronom renvoie à « Mmes de Pomponne et de Vins » (note 2 de la p. $728:$ II, 1438).

${ }^{9} \mathrm{R}$. Duchêne indique que le pronom réfère à Charles de Sévigné, et signale que dans l'édition Perrin (P2), il avait été remplacé par Mon fils (note 2 de la p. 844 : II, 1488).

${ }^{10}$ R. Duchêne explique le choix du pronom par un "accord selon l'idée », l'anaphore désignant «les amies et éventuellement leurs maris » (note 6 de la p. 872, III : 1563).

${ }^{11}$ R. Duchêne propose comme glose « aux femmes en général et aux belles en particulier » (note 10 de la p. 877 : III, 1566).

${ }^{12}$ Telle est la position de Vaugelas, Remarques sur la langue françoise utiles à ceux qui veulent bien parler et bien escrire, Paris, Veuve Jean Camusat et Pierre Le Petit, 1647 [Genève, Slatkine Reprints, éd. J. Streicher, 1934], p. 585.

${ }^{13}$ N. Fournier, Grammaire du Français classique, Paris, Belin, coll. "Lettres Sup », 1998, p. 178. Pour une synthèse des prises de position en faveur de l' "exigence de proximité ", voir G. Siouffi, Le "génie de la langue française" à l'âge classique. Recherches sur les structures imaginaires de la description linguistique de Vaugelas à Bouhours, thèse de doctorat, sous la dir. de P. Cahné, 2 volumes, Université de Paris IV-Sorbonne, janvier 1995, p. $105-112$.

${ }^{14}$ A. Jaubert, «La représentation pronominale. Contours et entours de la référence », L'Information grammaticale, $\mathrm{n}^{\circ} 82$, juin 1999 , p. 29-37.

${ }^{15}$ Dans la note 1 de la p. 712, R. Duchêne précise que le pronom renvoie à Mme de La Fayette (II, 1432).

${ }^{16}$ J.-M. Reichler-Béguelin, « Norme et textualité. Les procédés référentiels considérés comme déviants en langue écrite », dans G. Schoeni, J.-P. Bronckart et Ph. Perrenoud (dir.), La Langue française est-elle gouvernable ?, op. cit., p. 185-216.

${ }^{17}$ N. Fournier, Grammaire du français classique, op. cit., § 239, p. 178.

${ }^{18}$ G. Siouffi, «Le regard des grammairiens », dans B. Combettes (dir.), Évolution et variation en français préclassique. Études de syntaxe, Paris, Champion, 2003, p. 31-32.

${ }^{19}$ Ibid., p. 37.

${ }^{20}$ N. Fournier, Grammaire du français classique, op. cit., § 239-242, p. 178-180.

${ }^{21}$ Ibid., § 243-246, p. 180-182.

${ }^{22}$ I. Landy-Houillon, "Entre lexique et syntaxe : des interférences de la phrase et du pronom au XVII ${ }^{\mathrm{e}}$ siècle », dans M. Gross (dir.), La Lingua francese nel Seicento, Bari-Paris, Adriatica-Nizet, 1989, p. 63-78.

${ }^{23}$ I. Landy-Houillon rappelle opportunément que Mme de Sévigné « aimait chez Voiture certains "vicieux rapports" relevés par la censure d'Andry, mais sereinement assumés par leur auteur, ainsi que le montre le postscriptum d'une lettre à Costar » (ibid., p. 77).

${ }^{24}$ Ibid., p. 77-78.

${ }^{25}$ N. Fournier, Grammaire du français classique, op. cit., § 265, p. 190.

${ }^{26}$ Fr. Corblin, Les Formes de reprise dans le discours. Anaphores et chaînes de référence, Rennes, P.U.R., 1995.

${ }^{27}$ N. Fournier, Grammaire du français classique, op. cit., § 239, p. 178.

${ }^{28}$ Ibid., p. 182.

${ }^{29}$ N. Fournier, ibid., § 246, p. 181-182. Pour une présentation approfondie du divorce, à l'époque de Mme de Sévigné, entre la pratique anaphorique qui mise sur la saillance topicale d'un référent, et les injonctions normatives qui prônent le principe de proximité, voir du même auteur: "Norme et usage de l'anaphore pronominale en français classique : principe de proximité et principe de saillance du référent », dans J. Baudry et Ph. Caron (dir.), Problèmes de cohésion syntaxique, Limoges, P.U.LIM., 1998, p. 191-214.

30 Nous reprenons la métaphore suggestive qu'emploient W. Ayres-Bennett et $\mathrm{Ph}$. Caron dans leur «Introduction» aux Remarques de l'Académie française sur le Quinte-Curce de Vaugelas, Contribution à une histoire de la norme grammaticale et rhétorique en France, Paris, Presses de l'École normale supérieure, coll. «Études \& Documents en Histoire de la langue française », 1996, p. 40. 


\begin{abstract}
${ }^{31}$ Sur les enjeux de la discordance entre les usages de la référence pronominale et les normes en voie de constitution, voir N. Fournier, "Quelques problèmes concernant l'anaphore et les marqueurs anaphoriques dans Nicomède », L'Information grammaticale, $\mathrm{n}^{\circ} 76$, janvier 1998, p. 25-28.

${ }^{32}$ E. Gülich et Th. Kotschi, "Les marqueurs de la reformulation paraphrastique », Cahiers de linguistique française, $\mathrm{n}^{\circ} 5,1983$, p. 305-351. Sur le fonctionnement de la locution c'est-à-dire comme «instrument d'une fonction dialogique » visant à satisfaire le «besoin d'inter-locution et d'inter-compréhension », voir M. Murat et B. Cartier-Bresson, « C'est-à-dire ou la reprise interprétative », Langue française, n 73, février 1987, p. 5-15.

${ }^{33}$ Sur la manière dont Féraud blâme ce «commentaire métalinguistique », que Mme de Sévigné formule afin d'éclairer la construction elliptique qui précède, et sur « la valeur de cette retouche autonymique de l'épistolière qui, reformulant le sens au moyen d'une répétition, désamorce par avance les réserves d'une exégèse puriste en lui empruntant sa propre démarche », voir I. Landy-Houillon, "L'ellipse : une figure chez Mme de Sévigné ", Littératures classiques, $\mathrm{n}^{\circ}$ 28, automne 1996, p. 292.
\end{abstract}

